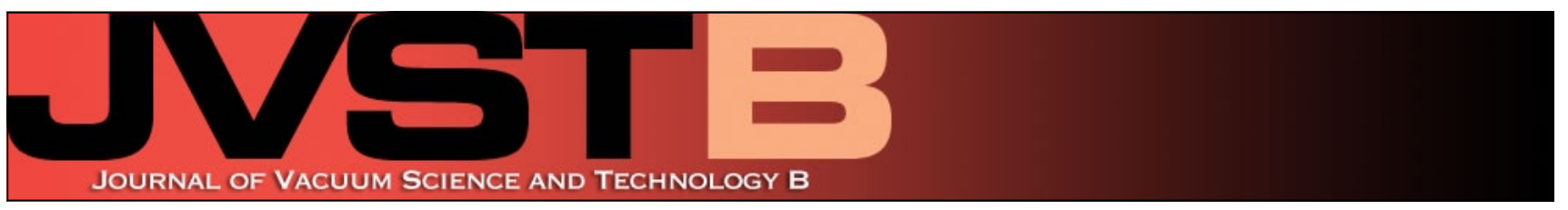

\title{
First-principles study of field-emission from carbon nanotubes in the presence of methane
}

Ali Kashefian Naieni, Parham Yaghoobi, and Alireza Nojeh

Citation: J. Vac. Sci. Technol. B 30, 021803 (2012); doi: 10.1116/1.3684980

View online: http://dx.doi.org/10.1116/1.3684980

View Table of Contents: http://avspublications.org/resource/1/JVTBD9/v30/i2

Published by the AVS: Science \& Technology of Materials, Interfaces, and Processing

\section{Related Articles}

Observation of fringelike electron emission pattern from triode Pt nano electron source fabricated by electronbeam-induced deposition

J. Vac. Sci. Technol. B 31, 02B107 (2013)

$\mathrm{X}$-ray tube with a graphite field emitter inflamed at high temperature

J. Vac. Sci. Technol. B 31, 02B106 (2013)

Improved approach to Fowler-Nordheim plot analysis

J. Vac. Sci. Technol. B 31, 02B103 (2013)

Illustrating field emission theory by using Lauritsen plots of transmission probability and barrier strength J. Vac. Sci. Technol. B 31, 02B102 (2013)

Experimental study of electric field screening by the proximity of two carbon fiber cathodes

J. Vac. Sci. Technol. B 30, 061803 (2012)

\section{Additional information on J. Vac. Sci. Technol. B}

Journal Homepage: http://avspublications.org/jvstb

Journal Information: http://avspublications.org/jvstb/about/about_the_journal

Top downloads: http://avspublications.org/jvstb/top_20_most_downloaded

Information for Authors: http://avspublications.org/jvstb/authors/information_for_contributors

\section{ADVERTISEMENT}

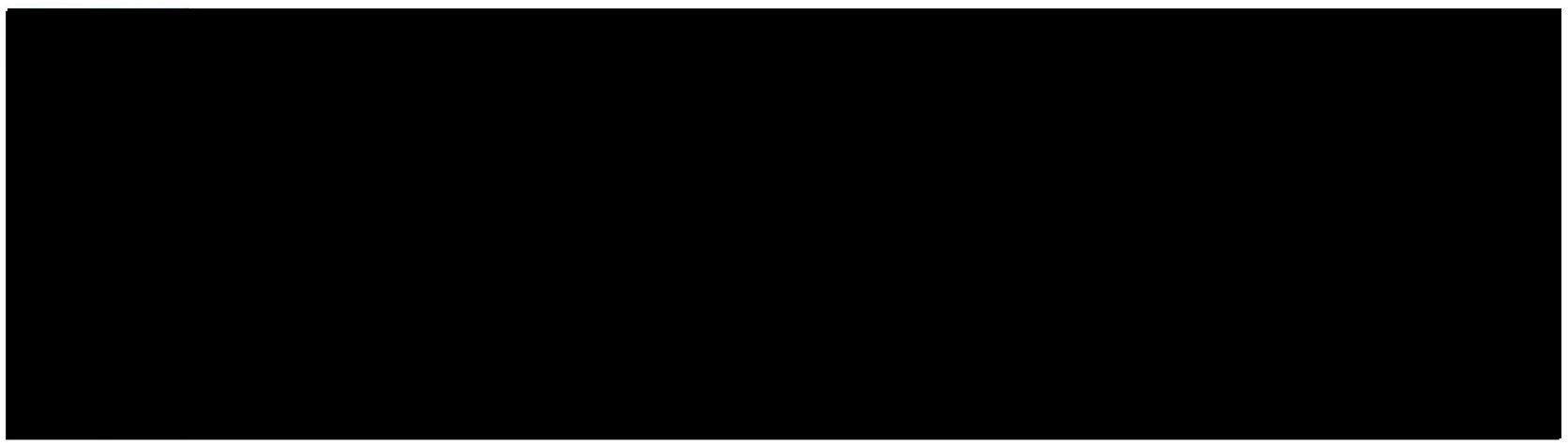




\title{
First-principles study of field-emission from carbon nanotubes in the presence of methane
}

\author{
Ali Kashefian Naieni, Parham Yaghoobi, and Alireza Nojeh ${ }^{\text {a) }}$ \\ Department of Electrical and Computer Engineering, The University of British Columbia, Vancouver, \\ British Columbia, V6T 1Z4, Canada
}

(Received 9 September 2011; accepted 26 January 2012; published 17 February 2012)

Carbon nanotubes are promising candidates for field-emitters. It has been shown that the presence of various gases can enhance or degrade the performance of nanotube emitters. Small hydrocarbons are of particular interest because of their ability to enhance the emission properties. The authors report a simulation study of field-emission from a carbon nanotube exposed to methane in various configurations with an emphasis on calculating the emission current. The Hartree-Fock theory combined with a Green's functions approach was used for the simulations. It was observed that the change in the emission current strongly depends on the particular arrangement of the methane molecules on the nanotube. (C) 2012 American Vacuum Society. [DOI: 10.1116/1.3684980]

\section{INTRODUCTION}

Using carbon nanotubes (CNTs) as electron field-emitters has attracted significant attention during the past decade and a half. ${ }^{1}$ Their promising properties such as mechanically strong and chemically stable structure, ability to carry very high current densities, and high aspect ratio make them excellent candidates for this area of application, although challenges exist on the way to their widespread practical usage.

The effect of various gas adsorbates on field-emission from CNTs has been investigated experimentally. ${ }^{2-7}$ For example, while oxygen reduces the emission current, ${ }^{2}$ water or small carbon-containing gases (methane and ethane) can enhance the emission characteristics. ${ }^{6,7}$

A number of simulation works have attempted to explain the experimental results. Maiti et al., ${ }^{8}$ Grujicic et al., ${ }^{9}$ and $\mathrm{Li}$ and Wang $^{10}$ used the ionization potential of the total structure including the CNT and adsorbates as a measure of the ability to emit electrons. Kim et al. ${ }^{11,12}$ included the local density of states at the CNT tip and gas adsorbates in their analysis. These studies have provided valuable insight. Nonetheless, not considering other important effects such as the change in the field-enhancement factor or emission sites due to the adsorbates have resulted in disagreements between the predictions of the simulation and the experimental results in some cases such as for methane: Experiments have shown an increase in emission current and a decrease in turn-on voltage in the presence of methane, ${ }^{4-6}$ whereas the first-principles simulations in Ref. 10 have shown that the ionization potential increases for a CNT structure with a methane molecule at the tip compared to a pristine CNT.

Here, we report a simulation study of the field-emission current of a CNT with various cases of methane adsorption on the tip. We used molecular dynamics (MD) simulations to find the different physically viable configurations of a $(5,5)$ CNT with zero to three methane molecules on its tip. First-principles simulations were then used to find the elec-

$\overline{{ }^{a} \text { Electronic mail: anojeh@ece.ubc.ca }}$ tronic structure. Building up on the results of these simulations and using a Green's functions approach, we then calculated the transmission probability and current-voltage characteristics for each case.

\section{METHODOLOGY}

An eight-unit-cell $(5,5)$ nanotube capped with half of a C60 molecule was used (Fig. 1). The dangling bonds on the other side of the CNT were eliminated using hydrogen atoms. Zero to three methane molecules were added onto the tip of the CNT. The structures were relaxed using MD simulations with adaptive intermolecular reactive empirical bond order potentials. ${ }^{13}$ These potentials are widely used for modeling intermolecular interactions in hydrocarbon sets. The MD simulations were performed using NANOHIVE-1 software $^{14}$ at 0.1 and $1 \mathrm{~K}$ of temperature. The structures were further relaxed with GAUSSIAN 09 software, ${ }^{15}$ using the restricted Hartree-Fock (HF) method with the 6-31 G(d) basis set. Structural relaxation was even performed in the case of the bare nanotube for consistency. The same method was used to simulate the electronic properties of the relaxed structures at each value of electric field. Note that in experiments, the electric field around the CNT tip is significantly enhanced compared to the macroscopic electric field between the electrodes (defined as voltage difference divided by the distance between the electrodes). Due to the limitation in the number of atoms in first-principles simulations and thus the short length of the nanotube used here, this field enhancement effect could not be captured by the simulation. Therefore, in the simulations we used electric field values that were on the order of the actual field around the CNT tip during field-emission (after taking the field enhancement effect into account). We will call this electric field the applied electric field from now on. However, it is important to note that the actual field around the nanotube is not uniform and is severely modified by the charge distribution on the nanotube itself in response to the applied electric field. The simulations took this effect into account through the self-consistent nature of the method used by calculating the charge distribution around the nanotube. Thus, the resulting, 


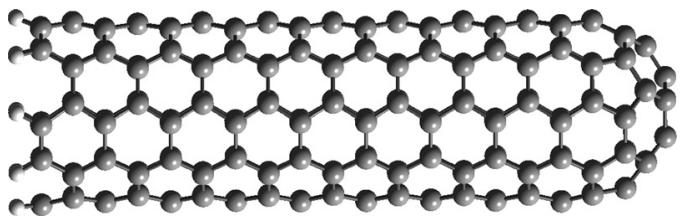

FIG. 1. Relaxed $(5,5)$ nanotube capped with half of a C60 molecule. The hydrogen atoms are used to eliminate the dangling bonds at the open end.

nonuniform field distribution that is due to both the applied, uniform electric field and the field arising from the response of the CNT was fully taken into account. The electrostatic potential distribution around the CNT, including $15 \mathrm{~nm}$ of free space in front of the CNT tip and excluding the hydrogen atoms at the base of the CNT and the four rows of carbon atoms immediately adjacent to them, was extracted at each field value (Fig. 2). The field-emission problem consists of electronic transport through the nanotube, into the adsorbate(s) and finally through the vacuum barrier. To solve for the emission current, rather than considering these transport problems separately, we used a method that we have previously devised and used for a bare nanotube. ${ }^{16}$ In this approach, the entire system including the CNT, adsorbate(s) and vacuum barrier (the region inside the cube shown in Fig. 2) constituted one device, sandwiched between two contacts (the cathode and anode electrodes). Electronic transport through the device (including the vacuum section) was thus simulated all at once by creating a threedimensional, real space Hamiltonian for the entire system using the potential profile extracted from the first-principles simulations. The Hamiltonian was used in a nonequilibrium Green's functions approach to find the transmission probability of electrons through the entire device at different energies for each value of the electric field. The tunneling currents at different fields at room temperature were calculated using the Landaur-Buttiker formula

$$
I=\frac{2 q}{h} \int T(E)\left[f_{1}(E)-f_{2}(E)\right] d E,
$$

in which $f_{1}(E)$ and $f_{2}(E)$ are the Fermi distribution functions of the electrodes on each side at room temperature, $T(E)$ is the electron transmission probability through the device at energy $E, q$ is the electron charge, and $h$ is Planck's constant.

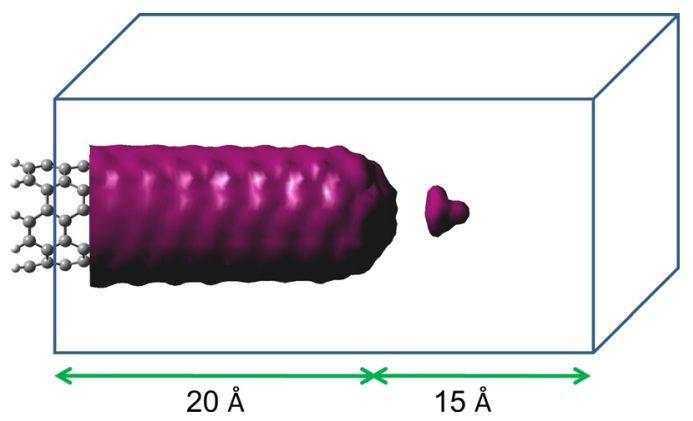

FIG. 2. (Color online) Simulated device, which contains the CNT, adsorbate(s) and the vacuum region. The purple surface shows a contour of the electrostatic potential profile around the CNT.
The details of this method have been reported previously. ${ }^{16}$ The same transport solver has also been used for the calculation of the field-emission current from CNTs under hydrogen adsorption ${ }^{17}$ and the study of electronic transport through deformed CNT structures. ${ }^{18}$

\section{RESULTS AND DISCUSSION}

We first discuss the case of one methane molecule on the nanotube tip. The final results of the MD simulation depend on the initial position and orientation of the methane molecule. In the first simulation, the methane molecule was placed such that the distance between the hydrogen atoms of its base to the CNT tip was $2.2 \AA$. During the simulation the methane molecule was repelled from the CNT to a $3.1 \AA$ distance and remained stable at that position. The initial orientation of the methane molecule (the base or a hydrogen pointing toward the CNT) did not change the final result in this case. In another simulation, the methane molecule was placed at $1.2 \AA$ away from the CNT with its base toward the CNT. Relaxing the structure led to the decomposition of the methane molecule, and the resulting hydrogen and methyl segments made bonds with the carbon atoms of the CNT cap.

When there is more than one methane molecule on the tip, depending on the initial configuration, the final relaxed structure could be the result of the interaction of the methane molecules with each other, as well as with the CNT. We investigated two structures each including two methane molecules and one with three methane molecules.

It is worth mentioning that although the MD simulations were carried out at 0.1 and $1 \mathrm{~K}$, the strong $\mathrm{sp}^{2}$ bonding keeps the structure stable at much higher temperatures, including at room temperature where the transport simulations were performed. However, one has to consider the fact that electron scattering by the lattice can become more significant as temperature rises. Nonetheless, experimentally it has been shown that the electron mean free path for metallic singlewalled CNTs is on the order of a micrometer at room temperature, ${ }^{19,20}$ which is substantially longer than our simulated structure; therefore, we believe it is justified to use a relaxed structure for the calculation of electronic transport and neglect electron-phonon interactions in our simulations.

As mentioned before, all of the final structures were geometrically optimized further using GAUSSIAN 09. This changed the distances between the CNT and the adsorbates to some extent. For example, in the case of a single methane molecule on the CNT tip, the distance between them increased from 3.1 to $3.8 \AA$ after this additional relaxation, which is close to the value of $3.92 \AA$ (distance from CNT to the carbon atom of the methane molecule) reported in Ref. 10.

Figure 3 shows a section of the simulated structures including the CNT tip and the methane molecules. The first structure has a methane molecule placed symmetrically in front of the CNT [Fig. 3(a)]. In the second structure the methane molecule is decomposed as explained before [Fig. 3(b)]. Figure 3(c) shows the two-methane case-1 


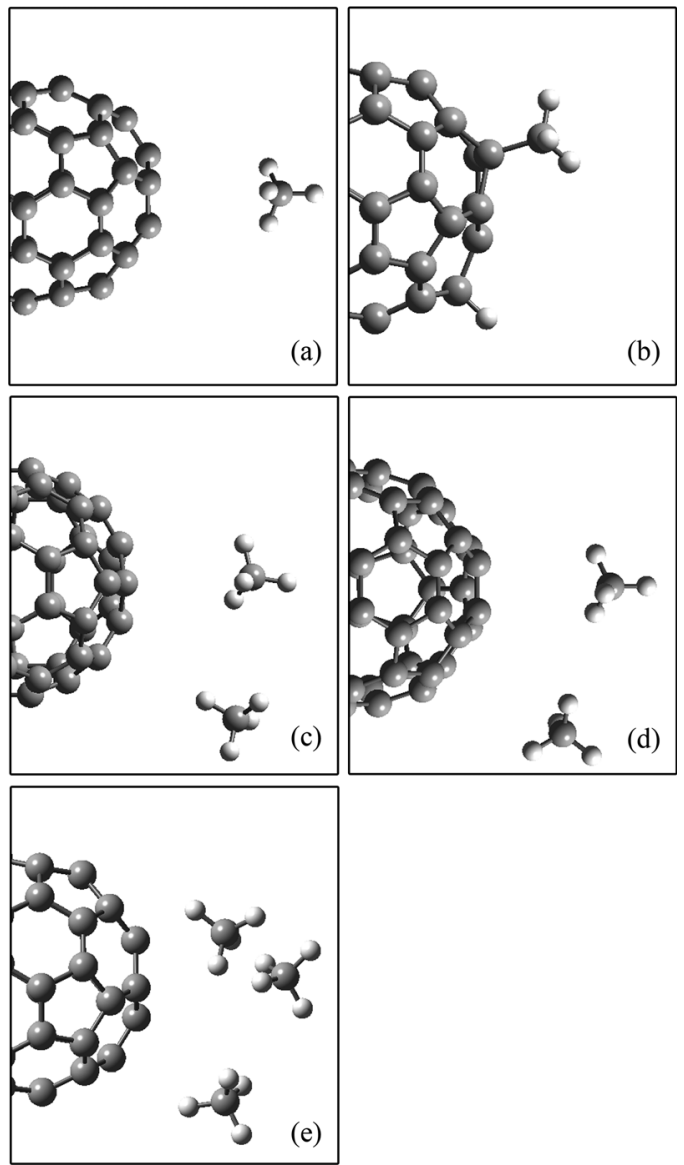

FIG. 3. Various simulated structures. The complete structures consist of a 200-atom capped (5,5) CNT as shown in Fig. 1, hydrogen atoms for eliminating the dangling bonds, and one or more methane molecules. The pictures here show only the tip of the CNT with (a) one methane molecule, (b) one methane molecule decomposed, (c) two methane molecules case 1, (d) two methane molecules case 2, (e) three methane molecules.

structure in which one methane molecule is pointing outward but the other one is not, whereas in the two-methane case- 2 structure both methane molecules are pointing outward [Fig. 3(d)]. In Fig. 3(e), the three-methane structure is shown in which the methane molecules have random orientations.

The simulations were performed for various electric field values up to $2 \mathrm{~V} / \AA$. An important question is whether these values are representative of the experimental reality, and whether the nanotube-adsorbate structure remains stable under such fields. The binding energy between the carbon atoms of a capped $(5,5)$ nanotube increases proportionally to the square of the magnitude of the electric field, ${ }^{21}$ which helps keep the structure intact even at high fields. Considering the large field enhancement of the order of thousands reported for small diameter CNTs, ${ }^{22-24}$ the upper limit of our simulations fall in the range of fields that are applied experimentally. For example, Bonard et $\mathrm{al}^{24}$ reported a field enhancement of up to 10000 for their samples. Considering the maximum voltage they had applied to their single-walled nanotube film $(\sim 4 \mathrm{~V} / \mu \mathrm{m})$, the maximum local field on the CNTs exceeds the maximum value applied in the present work.

Figure 4 shows the current versus electric field diagrams for all the cases. The bare-CNT result is presented on all of the graphs for ease of comparison. As it can be seen, the change in current as a result of methane adsorption is very different among the various cases. This shows that the fieldemission behavior highly depends on the type of interaction between the CNT and the methane molecules, as well as the exact configuration of the molecules around the CNT tip. Note that since the vertical axes are on a logarithmic scale, even small changes on these graphs represent significant current variations. In the case of a single methane molecule on the tip, the threshold voltage decreases and the current experiences an increase in low and very high fields compared to the case of the bare CNT [Fig. 4(a)]. In the decomposed methane case [Fig. 4(b)] and also when the two methane bases are toward the CNT [Fig. 4(d)], the current increase at low fields is even more pronounced. However, when the two methane molecules are pointing in different directions, the current experiences an overall decrease and the threshold field increases [Fig. 4(c)]. In the case of three methane molecules on the tip, a significant current decrease happens at high field values [Fig. 4(e)]. In this study, the threshold voltage was defined as the voltage at which the current reaches $10^{-20} \mathrm{~A}$. The reason for this choice was that this value of current is in the middle of the typical off-state current (around $10^{-30} \mathrm{~A}$ ) and on-state current (around $10^{-10} \mathrm{~A}$ ) on the log scale diagrams, and represents a region where the curve experiences a sharp jump from the off state to the on state. Although this choice may seem somewhat arbitrary, note that we use it only as a rough measure to compare the various curves and do not put emphasis on it as a rigorous definition of the threshold voltage.

As it can be inferred from Fig. 4, these diagrams do not follow a classic Fowler-Nordheim (FN) model in their entire range. ${ }^{25}$ The $\mathrm{FN}$ model is applicable to field-emission from a planar surface with a metallic density of states in which the electrons are accelerated toward a one-dimensional potential barrier. Although this model has been used for various fieldemitters and even for CNT emitters to various degrees, ${ }^{24,26}$ its strict applicability to such emitters is debatable. ${ }^{27} \mathrm{We}$ have previously discussed the discrepancies between the results of experimental and simulation reports on bare CNT emitters and the FN model, ${ }^{16}$ where we observed a non-FN and current saturation behavior that could be explained largely based on the manner in which the emission barrier at the nanotube tip evolves as the electric field increases. In particular, at low field values, the barrier decreases rapidly with increasing the electric field, whereas at high fields it becomes gradually less sensitive to the field value, leading to the saturation of the current.

In order to explain the changes to the field-emission behavior in various cases of methane adsorption, it should be noted that the methane molecules on the CNT tip not only change the electrostatic field distribution around the tip, but also modify the spots that contribute to electron emission, as well as the effective emission surface area. In previous simulation works, parameters such as ionization potential, work function, and position of the highest occupied molecular orbital (HOMO) have been used as a measure of how easily the electrons can reach the vacuum level. Another factor 

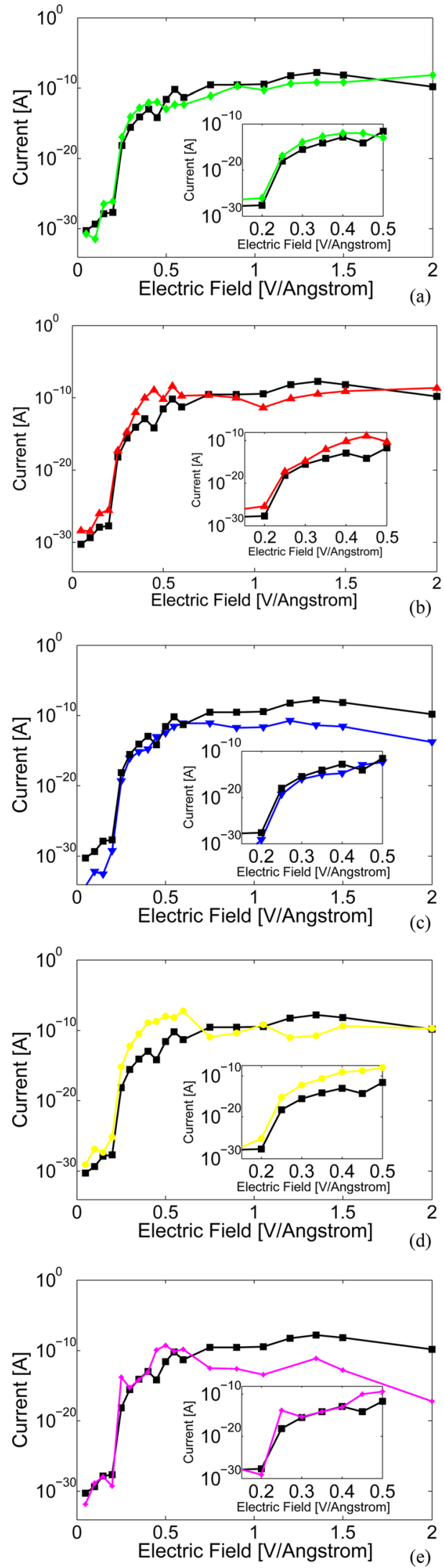

FIG. 4. (Color online) Current-field characteristics of the simulated structures: (a) one methane molecule, (b) one methane molecule decomposed, (c) two methane molecules case 1, (d) two methane molecules case 2, (e) three methane molecules, on the tip of the CNT. In all figures the curve for the bare CNT is also presented (black squares and line) for comparison. The insets show magnified views of the curves around the threshold. investigated has been the dipole moment, which could provide an indication of how the electric field and the emitter interact electrostatically. According to our simulations, the HOMO position of the structures at the smallest field value $(0.05 \mathrm{~V} / \mathrm{A})$ is $-5.68 \mathrm{eV}$ for the bare nanotube, $-5.59 \mathrm{eV}$ for the decomposed methane case, and very close to $-5.66 \mathrm{eV}$ for the rest of the cases. This suggests that overall the methane molecules facilitate the emission of electrons energetically. The smaller magnitude of changes in the HOMO energy compared to the results reported by Li et al. ${ }^{10}$ is due to the larger simulated structure in the present work (200 carbon atoms compared to 50), which reduces the sensitivity of the overall structure to the adsorbates. The dipole moments of the structures were investigated at various fields as well. At $0.3,0.75$, and $1.5 \mathrm{~V} / \AA$ field values, the dipole moment of the bare nanotube is $74.3,209.8$, and $516.2 \mathrm{D}$, respectively. The dipole moment of the nanotube with one methane molecule at the tip at the same field values is 75.2, 249.7, and $620.3 \mathrm{D}$, respectively. The increased dipole moments show that the methane molecule can significantly affect the interaction between the electrostatic field and the structure. However, despite the general insight that the behavior of HOMO, dipole moment, or other parameters can offer, as mentioned in the Introduction, there is no direct correlation between any single one of these parameters and the emission current at various fields for different structures with one or more methane molecules; these various parameters combine in complex ways to affect the behavior of the current. Therefore, an actual calculation of the emission current that automatically takes into account the combined behavior of these various effects is necessary, which is the topic of the present work. As such, the rest of the discussion will attempt to shed further light on the emission current behavior by taking a broader view, rather than singling out any one effect as the main mechanism behind the observed trends.

When the methane molecules are pointing outward from the CNT tip, the field enhancement factor is higher than that of the bare CNT, and the charge transfer and accumulation on the methane molecule facilitate the emission process. On the other hand, the number of atoms contributing to emission decreases compared to a bare CNT. Figure 5 shows the change in the charge distribution (the Mulliken charge population is shown) around the CNT tip at three different field values for the bare CNT and also when there is one methane molecule on the tip.

In the absence of adsorbates [Figs. 5(a)-5(c)], the electrons move toward the tip and accumulate on the very end of the CNT as the field increases. At the highest field shown in Fig. 5, a few carbon atoms at the tip have a charge of more than 0.187 electrons each. When there is a methane molecule on the tip, the charge on its carbon atom is 0.646 electrons under a field strength of $0.75 \mathrm{~V} / \AA$, significantly higher than the charge on any carbon atom in the bare CNT even at the highest field. This suggests that the carbon atom of the methane molecule will emit more effectively than any of the carbon atoms of the nanotube. On the other hand, the overall emission area is less than that of the bare nanotube, where several carbon atoms contribute to emission. The charge on 

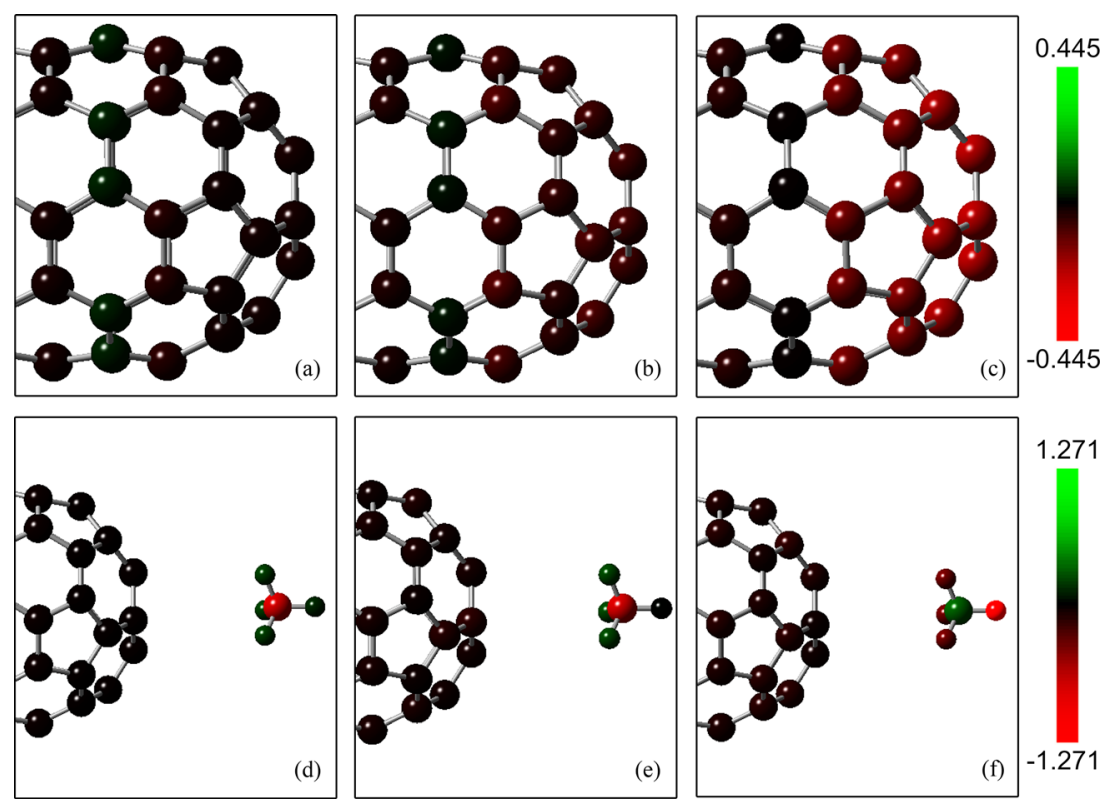

FIG. 5. (Color online) Mulliken charge population in a bare CNT [(a)-(c)] and when there is a methane molecule on the CNT tip [(d)-(f)] under electric field strengths of $0.3 \mathrm{~V} / \AA$ [(a),(d)], $0.75 \mathrm{~V} / \AA$ [(b),(e)], and $1.5 \mathrm{~V} / \AA$ [(c),(f)].

the hydrogen atom at the tip of the methane molecule (farthest from the nanotube) at $0.75 \mathrm{~V} / \AA$ is almost zero. As the field increases, the most highly charged point moves toward this hydrogen atom and emission happens mostly from this site. The charge on this hydrogen atom at a field strength of $1.5 \mathrm{~V} / \AA$ is 1.271 electrons while the carbon atom is charged positively to a value of 0.289 electrons. The hydrogen atom will thus constitute the main emission spot at high field strengths. Later we will discuss the emission spots further by examining the spatial distribution of the molecular orbitals.

Figure 6 shows the Mulliken charge distribution in the various structures at $1.5 \mathrm{~V} / \AA$. In the "two methane molecules case 1" [Fig. 6(c)] and "three methane molecules case" [Fig. 6(e)], the charge on the outermost hydrogen atom is significantly less than when the methane molecule is directly pointing outwards [Fig. 6(a)]. Consequently, emission is not as favorable in those cases, which shows itself as a decrease in current at higher fields [see Figs. 4(c) and 4(e)]. For the decomposed methane case [Fig. 6(b)], the charge accumulation on the resulting methyl group and hydrogen atom is not as significant as in the case of the intact methane molecule pointing outwards, but the effective emission area is increased.

Another way of evaluating the effectiveness of various atoms in emission and finding the emission sites consists of examining the molecular orbitals and their spatial distribution. Although emission is a result of contributions from all of the occupied orbitals, the ones with energies closest to the Fermi level play the most important role. The electron transmission probability is highest at energies very close to HOMO. Figure 7 shows HOMO for the case of a single methane molecule on the CNT tip at two different field values: HOMO mainly covers the area around the carbon atom of the methane molecule at $0.75 \mathrm{~V} / \AA$, whereas at $1.5 \mathrm{~V} / \AA$ the hydrogen atom experiences a much stronger coverage by the HOMO cloud. This
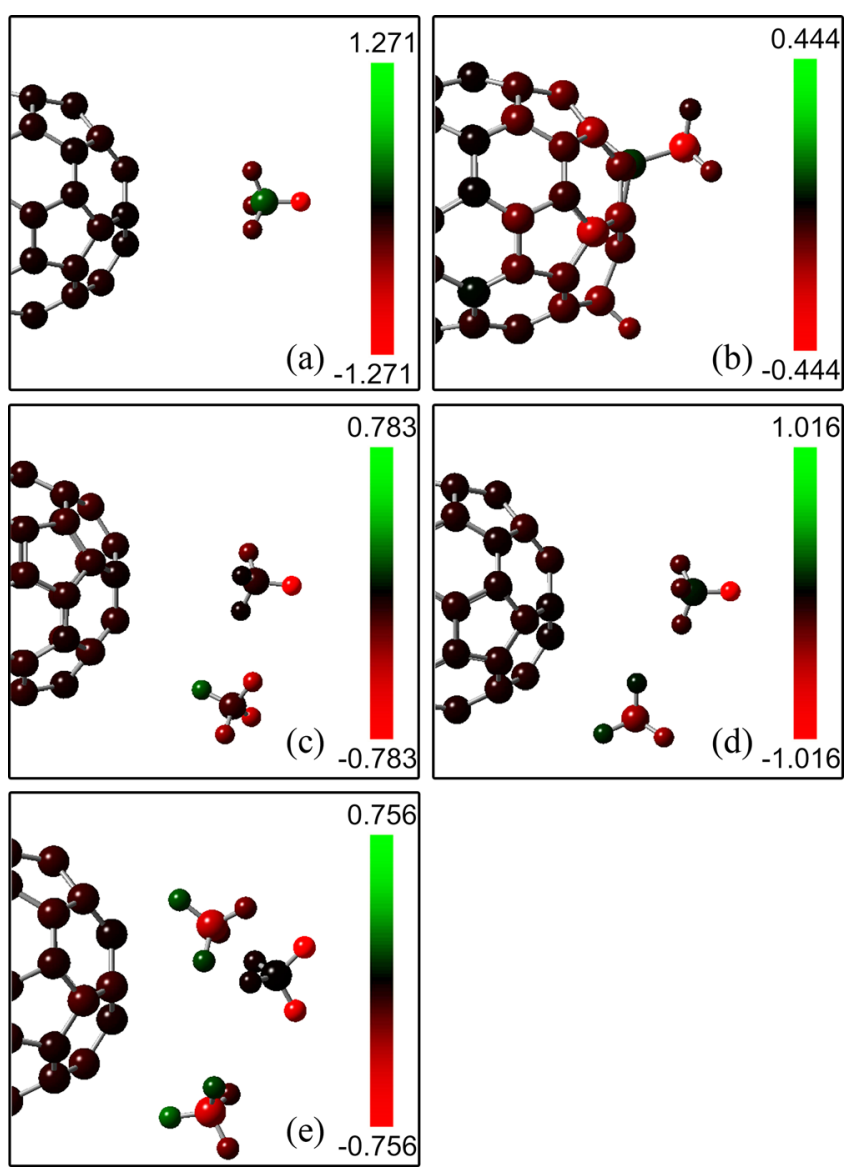

FIG. 6. (Color online) Mulliken charge distribution in various structures at $1.5 \mathrm{~V} / \AA$ : (a) one methane molecule, (b) one methane molecule decomposed, (c) two methane molecules case 1, (d) two methane molecules case 2, (e) three methane molecules, on the tip of the CNT. 

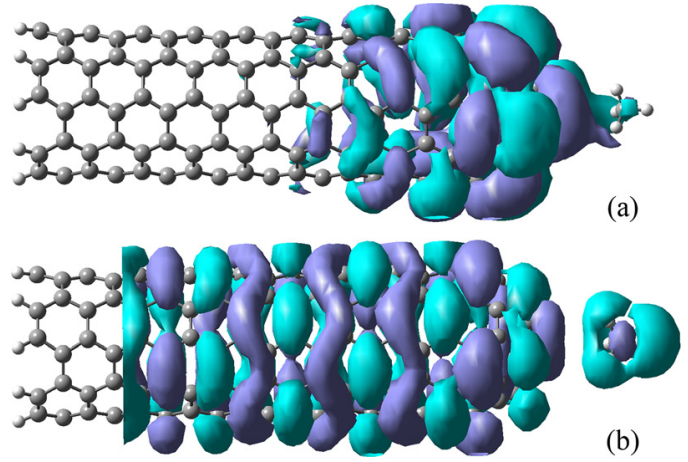

FIG. 7. (Color online) Spatial distribution of HOMO for the CNT with a single methane molecule on the tip under field strengths of $0.75 \mathrm{~V} / \mathrm{A}$ (a) and $1.5 \mathrm{~V} / \AA$ (b). The surfaces shown correspond to a contour value of 0.004 . The two distinct colors show the positive and negative lobes of the orbital.

figure thus provides insight into the changes in the fieldemission pattern as the applied field becomes stronger.

Figure 8 shows HOMO for the "two methane molecules case 1" and "two methane molecules case 2." We see that HOMO tends to cover the methane atoms further as the field strength increases. This happens at lower fields for the "two methane molecules case 2" [Fig. 8(e)] compared to "two methane molecules case 1" [Fig. 8(b)], indicating that the effect of the methane molecules is not quite the same even in structures with high similarity, and that the shape of the orbitals and consequently the emission current is a function of the precise arrangement of the methane molecules. Methane molecules pointing straight out from the nanotube (i.e., the "one methane molecule case" and "two methane molecules case 2 ") increase the field enhancement and the emission current more significantly at lower fields [see Figs. 4(a) and 4(d)].

Although the emission current is made of contributions from many orbitals, if one of these has a dominant effect, the field-emission spot is expected to have a shape similar to the spatial distribution of that orbital. Figure 9 shows an example where the front view (along the nanotube axis) of the spatial distribution of HOMO is seen for the "one methane molecule case." The distribution highlighted in red is reminiscent of the field-emission pattern of a multiwalled CNT after exposure to methane as reported by Saito (Fig. 11 of Ref. 28).

The experimental reports on field-emission of CNTs exposed to methane show a decrease in threshold voltage and increase in emission current. Given that, according to our simulation results described above, various configurations of methane molecules on the tip could increase or decrease the emission current at different field levels, the experimental observations are likely due to the aggregate effects of multiple CNTs with varying degrees of methane adsorption emitting at the same time, averaging out the effect of each emitter. Another important point is that the various cases of adsorption do not have the same probability of occurrence and, depending on their binding energy and stability, some cases may be dominant. We investigated the adsorption energy of the methane molecules on the CNT. The adsorption energy for the cases where the methane molecules were physisorbed was calculated using the following formula:

$$
E_{\mathrm{ads}}=E_{\mathrm{CNT}}+E_{\text {methane }}-E_{\mathrm{CNT}+\text { methane }} \text {. }
$$

The values found using the $a b$ initio simulations with no external field were used. The results for the single methane molecule, two methane molecules case 1, two methane molecules case 2, and three methane molecules were 0.038 , $0.025,0.024$, and $0.021 \mathrm{eV}$ per methane molecule, respectively. As the number of methane molecules on the nanotube increases, the binding energy of each molecule decreases and the molecules become less stable on the CNT.

The adsorption energies of methane molecules under $0.75 \mathrm{~V} / \AA$ were calculated as well and were 2.339, 0.617, 1.474 , and $1.183 \mathrm{eV}$ per methane for single methane molecule, two methane molecules case 1, two methane molecules case 2, and three methane molecules, respectively. These numbers were calculated using the following formula:
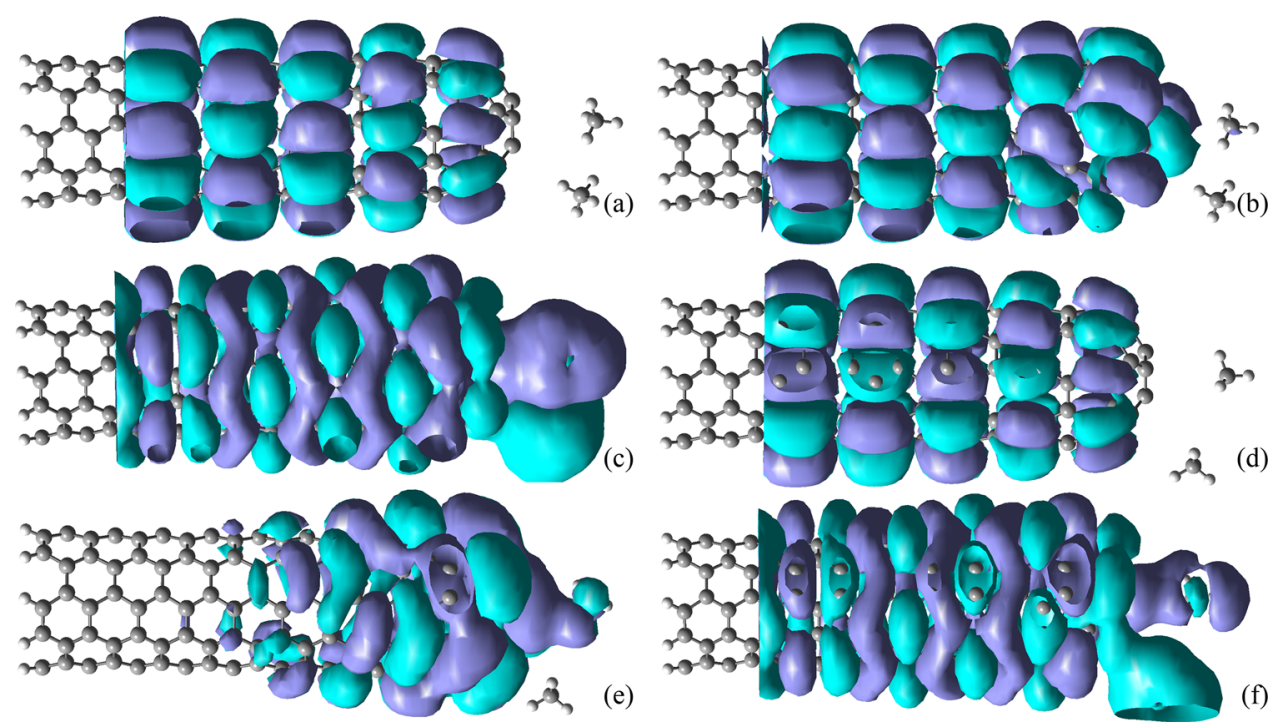

FIG. 8. (Color online) Spatial distribution of HOMO of the "two methane molecules case 1" [(a)-(c)] and "two methane molecules case 2" [(d)-(f)] for 0.3, 0.75 , and $1.5 \mathrm{~V} / \AA$, respectively. The surfaces shown correspond to a contour value of 0.002 . 


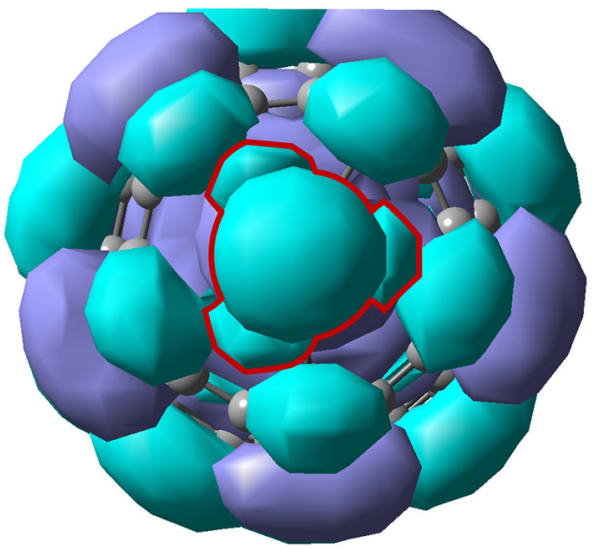

FIG. 9. (Color online) Spatial distribution of HOMO, viewed along the nanotube axis, when there is a methane molecule at the tip, under an electric field of $1.5 \mathrm{~V} / \AA$ (contour value $=0.005$ ). The parts due to the methane molecule are outlined in red.

$$
\begin{aligned}
E_{\mathrm{ads}}(\text { under }- \text { field })= & E_{\mathrm{CNT}}(\text { under }- \text { field }) \\
& +E_{\text {methane }}(\text { no }- \text { field }) \\
& -E_{\mathrm{CNT}+\text { methane }}(\text { under }- \text { field }) .
\end{aligned}
$$

This is the energy required to detach the methane molecule(s) and take it/them far away from the tip where the electrostatic field is negligible compared to the field around the tip. We see that the adsorption energy of the methane molecules increases considerably under the field compared to the no field scenario, which keeps the structure stable even at high fields. The structures with higher symmetry have a larger adsorption energy compared to the other cases. The single methane case has the highest adsorption energy under the field. Therefore, in a collection of nanotube emitters, one expects to see a larger number of nanotubes with one methane molecule, corresponding to the current-field characteristics shown in Fig. 4(a) where the threshold voltage is reduced and emission current is increased at low fields, consistent with experimental observations.

\section{SUMMARY}

A Green's functions approach, combined with firstprinciples simulations, was used to calculate the field-emission current from a CNT with methane molecules adsorbed on its tip. Various cases including CNT with no methane, one methane molecule physisorbed, one methane molecule chemisorbed, two configurations of two methane molecules with different arrangements, and one configuration including three methane molecules were investigated. The results show that the emission current depends on the type of interaction between the CNT and the methane molecules (physisorption or chemisorption), as well as the exact arrangement of the methane molecules on the CNT. Overall, the current increases as a result of exposure to methane for the cases in which the methane molecule/molecules increase the field enhancement or the emission area. The charge population on the atoms and the spatial distribution of the molecular orbitals provided insight into the behavior of the emission current in the various cases. It was shown that the emission pattern changes and the atoms on the very end of the structure play a more significant role in emission as the applied field increases.

\section{ACKNOWLEDGMENTS}

The authors thank the Natural Sciences and Engineering Research Council (NSERC) and the BCFRST Foundation/ British Columbia Innovation Council for financial support. This research has been enabled in part through the use of advanced computing resources provided by WestGrid and Compute/Calcul Canada.

${ }^{1}$ Carbon Nanotube and Related Field Emitters: Fundamentals and Applications, edited by Y. Saito (Wiley-VCH Verlag, Weinheim, 2010).

${ }^{2}$ A. Wadhawan, R. E. Stallcup, K. F. Stephens, J. M. Perez, and I. A. Akwani, Appl. Phys. Lett. 79, 1867 (2001).

${ }^{3}$ K. S. Yeong and J. T. L. Thong, Appl. Surf. Sci. 233, 20 (2004).

${ }^{4}$ A. M. Fennimore, D. H. Roach, G. A. Wilson, F. M. Pellicone, and L. T. Cheng, Appl. Phys. Lett. 92, 213108 (2008).

${ }^{5}$ L. M. Sheng, P. Liu, Y. M. Liu, L. Qian, Y. S. Huang, L. Liu, and S. S. Fan, J. Vac. Sci. Technol. A 21, 1202 (2003).

${ }^{6}$ H. Nakahara, T. Yamashita, and Y. Saito, e-J. Surf. Sci. Nanotechnol. 8, 77 (2010).

${ }^{7}$ K. A. Dean, P. Von Allmen, and B. R. Chalamala, J. Vac. Sci. Technol. B 17, 1959 (1999).

${ }^{8}$ A. Maiti, J. Andzelm, N. Tanpipat, and P. von Allmen, Phys. Rev. Lett. 87, 155502 (2001).

${ }^{9}$ M. Grujicic, G. Cao, and B. Gersten, Appl. Surf. Sci. 206, 167 (2003).

${ }^{10} \mathrm{Z}$. Li and C. Y. Wang, Chem. Phys. 330, 417 (2006).

${ }^{11}$ C. Kim, Y. S. Choi, S. M. Lee, J. T. Park, B. Kim, and Y. H. Lee, J. Am. Chem. Soc. 124, 9906 (2002).

${ }^{12}$ C. Kim, K. Seo, B. Kim, N. Park, Y. S. Choi, K. A. Park, and Y. H. Lee, Phys. Rev. B 68, 115403 (2003).

${ }^{13}$ S. J. Stuart, A. B. Tutein, and J. A. Harrison, J. Chem. Phys. 112, 6472 (2000).

${ }^{14}$ B. Helfrich, NANOHIVE-1, v. 1.2.0-b1, Nanorex, Inc., 2005.

${ }^{15}$ M. J. Frisch et al., Gaussian09, Revision A. 02, Gaussian, Inc., Wallingford, CT, 2009.

${ }^{16}$ P. Yaghoobi, K. Walus, and A. Nojeh, Phys. Rev. B 80, 115422 (2009).

${ }^{17}$ P. Yaghoobi, M. K. Alam, K. Walus, and A. Nojeh, Appl. Phys. Lett. 95, 262102 (2009).

${ }^{18}$ A. Kashefian Naieni, P. Yaghoobi, D. J. Woodsworth, and A. Nojeh, J. Phys. D 44, 085402 (2011).

${ }^{19}$ M. S. Purewal, B. H. Hong, A. Ravi, B. Chandra, J. Hone, and P. Kim, Phys. Rev. Lett. 98, 186808 (2007).

${ }^{20}$ J. Park, S. Rosenblatt, Y. Yaish, V. Sazonova, H. Ustunel, S. Braing, T. A. Arias, P. W. Brouwer, and P. L. McEuen, Nano Lett. 4, 517 (2004).

${ }^{21}$ C. Kim, B. Kim, S. M. Lee, C. Jo, and Y. H. Lee, Phys. Rev. B 65, 165418 (2002).

${ }^{22}$ Z. Xu, X. D. Bai, and E. G. Wang, Appl. Phys. Lett. 88, 133107 (2006).

${ }^{23}$ J. Y. Huang, S. Chen, S. H. Jo, K. Kempa, and Z. F. Ren, Microsc. Microanal. 11, 1926 (2005).

${ }^{24}$ J. M. Bonard, J. P. Salvetat, T. Stockli, W. A. de Heer, L. Forro, and A. Chatelain, Appl. Phys. Lett 73, 918 (1998).

${ }^{25}$ R. H. Fowler, and L. Nordheim, Proc. R. Soc. London, Ser. A 119, 173 (1928).

${ }^{26}$ Z. Xu, X. D. Bai, E. G. Wang, and Z. L. Wang, Appl. Phys. Lett. 87, 163106 (2005)

${ }^{27}$ P. Yaghoobi and A. Nojeh, Mod. Phys. Lett. B 21, 1807 (2007).

${ }^{28}$ Y. Saito, Coord. Chem. Rev. 253, 2912 (2009). 